\title{
Autorégulation de rapports sociaux et dispositif dans Wikipedia
}

\author{
Bernard JACQUEMIN \\ Université de Haute Alace / CREM EA3476 \\ 16 rue de la Fonderie F-68 093 Mulhouse cedex (France) \\ Bernard.Jacquemin@ uha.fr
}

\begin{abstract}
Résumé
La nature collaborative de l'encyclopédie en ligne Wikipédia amène naturellement ses contributeurs à travailler avec d'autres et à confronter leurs idées et points de vue. Or ni les cinq principes fondateurs, ni le logiciel wiki utilisé comme support de l'encyclopédie ne déterminent un cadre à cette collaboration. Dans cet article, nous étudions les initiatives spontanées de la communauté des contributeurs pour favoriser la collaboration, les échanges sociaux et la résolution des conflits. Pour analyser ces démarches, nous exploitons la notion de dispositif, et en particulier le mode de mise en place d'une gouvernance dans un dispositif collaboratif. Nous remarquons une tension entre deux visions du pouvoir, l'une qui favorise la transgression moyennant une fécondité créatrice dans l'optique de l'objectif de Wikipédia, tandis que l'autre veille au strict respect du cadre du dispositif. Si la seconde position semble l'emporter, nous notons toutefois que le loyalisme est moins tangible qu'il n'y paraît. Mots-clefs : encyclopédie Wikipédia, dispositif collaboratif, gouvernance, controverse, conflit, pouvoir, transgression.

As a collaborative work, the online encyclopaedia Wikipedia leads naturally the contributors to work with each other and to face their opinions. But no frame is provided to control the collaboration, neither in the five fundamental principles, nor from the wiki software. This article studies how the contributing community thinks up original ways to promote collaboration, social exchanges and conflict resolution. The concept of device (dispositif), and especially how governance shows itself in a collaborative device, is used to analyse these ways. Two views of the power conflict in the community: one permits contributors to break the rules to strive to Wikipedia's goal; the other one makes sure to enforce strictly the rules. Even though the latter seems to prevail, there is some evidence that loyalty may sometimes be illusory.

Keywords: Wikipedia encyclopaedia, collaborative device, governance, dispute, conflict, power, violation.
\end{abstract}

\section{Introduction}

Le 15 janvier dernier, l'encyclopédie Wikipedia fêtait ses dix années d'existence, dix ans qui lui ont suffi pour devenir le point d'entrée de référence vers l'information encyclopédique dans tous les domaines de la connaissance, y compris ceux que les encyclopédies traditionnelles ne prennent pas en compte. En effet, Wikipedia est une initiative de collecte et de diffusion de connaissances universelles qui repose sur des principes parfois éloignés du fonctionnement éditorial historique de l'encyclopédie classique, alors même qu'une qualité scientifique équivalente demeure son objectif. Ainsi, Wikipedia désigne une initiative de construction collaborative de savoirs, pour laquelle tout un chacun peut se prévaloir d'une connaissance ou d'une expertise sans avoir à revendiquer un statut, et peut de ce fait endosser chacun des deux rôles du spécialiste, tant celui du savant appelé à rédiger tout ou partie d'un article dans son domaine de compétence que celui de l'expert chargé de critiquer et de valider les contenus issus du travail d'écriture. De manière générale, Wikipedia désigne à la fois la somme encyclopédique qui constitue le résultat de la mise en commun de cette masse de connaissances, et l'organisation communautaire qui assure le bon fonctionnement de cette collaboration et de cette diffusion.

En tant que projet collaboratif de mise en commun de connaissances pour la constitution d'une plateforme encyclopédique, et puisque cette plateforme représente à la fois le support technique de réalisation du projet et l'espace de communication et de réalisation consacré à cet objectif, Wikipedia constitue un bel exemple de dispositif collaboratif selon la lecture de Foucault qu'en fait Peraya (1999). En effet, exploitant les fonctionnalités idoines offertes par une plateforme technique, le projet Wikipedia offre aux participants un espace social propice à une collaboration et à une interaction établies dans l'optique de la finalité du projet luimême. Par exemple, Wikipedia repose sur cinq principes fondateurs immuables et simples qui régissent intégralement son fonctionnement scientifique et social et la mise en œuvre de la démarche de contrôle et d'amélioration qui la sous-tend, ce qui le rapproche de la vision de Foucault et al. (1994) de la gouvernance d'un dispositif. Au cours de son évolution, le projet Wikipedia s'est cependant vu confronté à une réalité et à des situations qui n'avaient pas été envisagées lors de sa création - notamment les contraintes liées à une collaboration pas toujours sereine - et qui, sortant du modèle socio-technique établi, ne bénéficiaient d'aucun cadre pour répondre à l'objectif de l'encyclopédie. Les contributeurs de Wikipedia ont donc progressivement intégré ces situations dans leurs habitudes, et ont souvent décidé de s'éloigner du cheminement standard de Certeau (1980); Hert (1999) pour proposer des conseils et des procédures permettant à la fois d'accompagner au mieux la démarche de collaboration à l'encyclopédie et de protéger cette dernière contre toute intervention malheureuse, qu'elle 
soit malveillante ou non. Ce faisant, ils ont débordé le cadre offert par le dispositif initial et proposé un nouveau cadrage qu'il s'est dès lors agi de légitimer.

Dans cet article, nous confrontons la notion théorique du dispositif avec une réalité collaborative de terrain. En particulier, nous étudions la démarche de la communauté collaborante pour légitimer diverses initiatives irrégulières, démarche à travers laquelle cette communauté construit perpétuellement un équilibre fragile entre les règles et principes qui fondent le dispositif et l'objectif qui justifie son existence. Nous nous penchons d'abord sur plusieurs de ces initiatives de la communauté des contributeurs à la Wikipédia francophone, ainsi que sur la question de leur légitimation. Après avoir décrit le projet encyclopédique dans son organisation initiale et théorique, nous nous intéressons en particulier à la manière dont le dispositif influence l'organisation de la collaboration de cette communauté. Nous étudions d'abord les espaces que les contributeurs utilisent ou se ménagent pour assurer les échanges, ensuite les procédures qui sont progressivement mises en place d'une part pour favoriser la collaboration, et de l'autre pour garantir la sérénité de ces échanges. Nous nous penchons notamment sur le processus de désignation des arbitres, qui mêle de manière inextricable des initiatives détachées du fonctionnement de Wikipédia et un loyalisme parfois supposé à ses principes. Nous verrons qu'en faisant spontanément émerger des espaces de liberté pour la communication et la collaboration, puis en les intégrant au dispositif sous forme de procédures plus ou moins strictes fournissant un cadre structuré à la collaboration, la communauté wikipédienne 11 montre une tension entre deux conceptions constitutives de la gouvernance au sein d'un dispositifl. Elle opte en définitive pour une application stricte de ses principes dans l'exercice du pouvoir, mais ces principes s'expriment parfois plus formellement qu'effectivement.

\section{Le projet Wikipedia}

D'une ampleur et d'une popularité inégalées 3 , il n'est plus besoin de présenter l'encyclopédie collaborative en ligne Wikipedia : fournissant plus de 17,6 millions d'articles répartis (inégalement) sur 277 langues, elle représente la démarche encyclopédique la plus ambitieuse et la plus aboutie à ce jour. Mais malgré la popularité de Wikipedia, les règles qui sous-tendent son activité sont relativement peu connues des usagers qui la consultent, et dont l'immense majorité ne sont pas contributeurs (près de $94 \%$ des inscrits, sans compter les usagers anonymes de l'encyclopédie, ne sont jamais intervenus dans la rédaction du moindre article 4 .
Pour assurer son fonctionnement, les créateurs de Wikipedia, Jimmy Wales et Larry Sanger, s'appuient sur deux éléments : d'abord sur un logiciel informatique appelé wiki, dont l'intérêt est qu'il permet l'édition rapide et facile d'une page web et sa mise en ligne immédiate ; ensuite sur cinq principes fondateurs immuables - ou piliers -, qui sont les dogmes auxquels tout participant au projet doit se conformer. Il s'agit des principes suivants : la pertinence encyclopédique, comprise ici comme une synthèse des connaissances sur un sujet précis désigné par le titre de l'article, de portée universelle, et déjà diffusées ou publiées (ce qui implique un sourçage); la neutralité de point de vue, principe wikipédien qui garantit une forme d'objectivité, puisqu'il s'agit de mentionner les différentes théories ou opinions en présence (avec des sources) sans prendre position pour l'un ou pour l'autre; la courtoisie, basée sur un principe d'égalité et de respect mutuel des contributeurs, et privilégiant le consensus; la liberté de droits liés aux informations qui y sont apportées 5 , conférant à chacun le droit de créer, copier, modifier et distribuer le contenu de l'encyclopédie avec les mêmes droits; le cinquième principe indique qu'aucune autre règle fixe n'existe en dehors des quatre précédentes. Rapidement, la version anglophone initiale s'est assortie d'instances comparables dans de nombreuses autres langues. Sous l'égide de la Wikimedia Foundation Inc., gestionnaire du projet initial et de ses dérivés, les nouvelles instances linguistiques de l'encyclopédie sont autonomes - et ne constituent donc pas des traductions de la version initiale mais elles reposent elles aussi sur un wiki et sur les cinq piliers fondateurs.

Les principes qui fondent le projet Wikipedia s'appuient sur plusieurs axiomes forts. Tout d'abord, il s'agit d'une encyclopédie collaborative dans laquelle chaque participant est susceptible d'être acteur en contribution ou en critique, sans qu'un contributeur ait plus de poids qu'un autre. Aucun contributeur ne peut donc se prévaloir d'un statut particulier pour asseoir sa position par rapport à une autre. En cela, Wikipedia est résolument démocratique, voire égalitariste. Ce positionnement est très sensible à travers toutes les instances et procédures qui peu à peu se sont mises en place pour une bonne gestion du projet. Ensuite, Wikipedia s'est développé en choisissant de mettre en œuvre le logiciel wiki non seulement parce qu'il permettait de disposer des fonctionnalités voulues pour faciliter une publication collaborative, mais aussi en raison des droits associés à la plateforme wiki, publiée sous licence libre GNU-GPL6. Cette philosophie héritée des communautés du logiciel Open Source est clairement perceptible à travers l'ensemble du projet, ne

1. La communauté des contributeurs à Wikipédia a forgé le nom commun « wikipédien », qui désigne les membres inscrits à l'encyclopédie Wikipédia. Sous sa forme adjectivale, il signifie « relatif à Wikipédia ». Nous jugeons ces néologismes pertinents et avons choisi de les utiliser dans cet article.

2. Notre objectif n'est pas d'étudier l'émergence d'une gouvernance au sein de Wikipedia, mise en évidence (entre autres) dans des travaux comme ceux de Barbe (2009) ou Levrel et Cardon 2009). Nous nous intéressons ici à la notion de dispositif et à la contrainte que cette notion exerce sur cette gouvernance.

3. Plusieurs statistiques de consultation des sites web les plus fréquentés, consultées le 18 mars 2011, font apparaître Wikipedia tantôt au $5^{\mathrm{e}}$ rang (http://www.google.com/adplanner/static/top1000/; classement n'intégrant pas Google, forcément premier), tantôt au $8^{\mathrm{e}}$ rang (http://www . alexa.com/topsites; classement intégrant Google).

4. http://stats.wikimedia.org/EN/TablesWikipediaZZ.htm

5. Creative Commons CC-BY-SA (paternité-partage des conditions initiales à l'identique), http://fr.wikipedia.org/wiki/Wikipédia:Droit_d'auteur

6. Licence fixant la plupart du temps les conditions légales de distribution des logiciels libres, http://www.gnu.org/licenses/gpl.html

7. Dans son acception politique et non économique. 
fût-ce que dans le principe fondateur imposant la liberté de droits pour l'information contenue et diffusée. Une coloration extrêmement libérale $7^{7}$ traverse donc l'ensemble du projet, concrétisée tantôt via une application directe de la consultation démocratique (où tout adhérent du projet est l'égal des autres et à ce titre peut faire des propositions pour l'évolution tant de la communauté que du contenu encyclopédique), tantôt à travers l'idéal de l'accès libre à un savoir universel qui serait la propriété de tous.

$\mathrm{Au}$ cours de l'évolution du projet, les différentes instances linguistiques, relativement indépendantes les unes des autres, ont vu leur autonomie s'affirmer. Bien que soumise aux principes fondateurs de Wikipedia, chaque version de l'encyclopédie a petit à petit adopté les habitudes, conseils et procédures mis en place par sa propre communauté contributrice, plus ou moins indépendamment des initiatives de ses homologues en d'autres langues. En cela, l'esprit démocratique de Wikipedia se manifeste, puisque l'ensemble des mesures adoptées le sont sur proposition de la communauté active dans l'instance concernée, et suite à un vote positif de ladite communauté. Dorénavant, les faits et phénomènes que nous observerons seront plus particulièrement issus de l'instance francophone de l'encyclopédie, appelée Wikipédia 8 , que nous étudions et comparons parfois à la version « originale » de l'encyclopédie, la Wikipedia anglophone initiale.

\section{Collaboration dienne}

\subsection{Initier la collaboration}

À ses débuts, la collaboration dans Wikipédia est fondée intégralement sur deux facteurs. Tout d'abord le fonctionnement pratique du logiciel wiki qui permet l'édition simple et rapide de pages web en ligne, qui associe également une page de discussion à chaque page web «principale », consacrée à un article encyclopédique, donnant ainsi la possibilité d'entretenir un lien social entre contributeurs; l'identification respective des collaborateurs est alors assurée soit par un identifiant librement choisi par le contributeur s'il décide de s'inscrire régulièrement, soit par son adresse IP s'il préfère rester anonyme. Ensuite, la philosophie générale des porteurs du projet, qui promeuvent ouvertement les valeurs démocratiques et la liberté juridique. Cet aspect démocratique et égalitaire des contributeurs suscite une autre forme de collaboration, plus critique que la simple construction plus ou moins simultanée d'une synthèse de connaissances sur un thème précis : en effet, tout contributeur peut endosser un rôle d'expert scientifique défavorable à l'encontre d'un article, et peut ainsi en modifier le contenu, sans forcément s'en expliquer dans le seul espace de contact prévu dans le wiki : la page de discussion. Ces deux facteurs sont matérialisés par l'utilisation du logiciel wiki, et par l'énonciation des cinq piliers fondateurs du projet.

Toutefois, cette collaboration wikipédienne originelle et la nécessité d'atteindre à la « neutralité de point de vue » a très tôt et naturellement engendré des désaccords entre contributeurs participant à un même article. Des mécanismes ont donc dû être envisagés pour protéger l'encyclopédie des dérives pouvant naître des confrontations d'idées et d'opinions, et pour régler au mieux les conflits qui parfois en découlent. Par ailleurs, dans sa perspective la plus dynamique et la plus constructive, l'objectif du projet reste bien entendu d'atteindre à un idéal informationnel dans tous les articles traités. Une série de mécanismes a ici aussi été inventée pour encourager les contributeurs à améliorer le fruit de leur travail pour atteindre à cet idéal.

\subsection{Favoriser la participation}

Les initiatives visant à encourager les contributeurs à enrichir toujours plus l'encyclopédie constituent la première catégorie des apports spontanés de la communauté au dispositif wikipédien, en dehors de toute structure préétablie. Dépassant les possibilités offertes par la maquette originale, la communauté a ainsi très rapidement proposé d'aider au fonctionnement de Wikipédia. Utilisant les possibilités classiques d'un éditeur de page web, la communauté s'est entendue pour signaler à l'intérieur même des articles les faiblesses particulières dont ils sont entachés. Ainsi, il n'est pas rare de trouver çà et là dans un article la mention d'une déficience de source, et appelant donc les contributeurs à citer davantage l'origine des informations qu'ils apportent.

Progressivement, une normalisation de ce type de mention a émergé, notamment à travers une échelle de dix-sept manquements concernant les sources d'information, allant du plus bénin (« référence souhaitée » pour permettre au lecteur de vérifier ou d'approfondir une information, qui par ailleurs n'est pas mise en doute) au plus grave (« article sans source » portant sur un article à l'information douteuse et controversée qui ne serait pas du tout étayée). Outre une mise en forme d'abord flottante, puis normalisée et donc très précise de chaque type de signalement de défaut, la communauté wikipédienne a créé un ensemble de pages dédiées non plus à la communication d'informations encyclopédiques, mais à la diffusion de conseils pour l'administration de l'encyclopédie. Accessibles depuis la page dédiée aux recommandations, plusieurs types de signalements ont ainsi été proposés par la communauté, par exemple « suspicion d'autobiographie »?

Toujours dans l'optique de favoriser la participation des contributeurs à l'enrichissement de Wikipédia, la communauté a imaginé l'adjonction de bandeaux en tête d'article. Il s'agit simplement, pour n'importe quel contributeur, de placer un balisage graphique et typographique - dont la forme et l'utilisation ont également été progressivement réglementées - immédiatement après le titre de l'article pour attirer l'attention de la communauté sur un problème ou sur un état lié à l'article concerné. Certains bandeaux sont informatifs, comme le bandeau « ébauche », qui invite la communauté à enrichir l'article concerné dont l'information actuelle est vraiment très lacunaire, ou le bandeau « sous protection », qui signale que le sujet traité a suscité une confrontation d'idées tellement passionnée qu'un administrateur élu par la communauté a dû blo-

8. Notez l'utilisation de l'accent aigu pour l'encyclopédie francophone Wikipédia, que nous opposons à la graphie sans accent Wikipedia, réservée ici au projet initial.

9. http://fr.wikipedia.org/wiki/Wikipédia:Recommandation 
quer les possibilités d'édition pour tempérer les protagonistes et protéger l'encyclopédie.

D'autres constituent des alertes qui signalent à la communauté que ses procédures de travail ont été transgressées dans le corps de l'article. En ce qui concerne les piliers fondateurs de Wikipédia, on trouve donc un bandeau signalant un " désaccord de pertinence » lorsque le sujet traité ne répond pas aux critères encyclopédiques définis par Wikipédia; une " controverse de neutralité » portant sur des sujets où le principe wikipédien de représentation de tous les points de vue n'est pas respecté; une violation du copyright, qui concerne la transgression du principe de liberté de droits liés à l'information mise en ligne. Une spécificité de l'usage du bandeau est que, s'il apparaît bel et bien dans l'article concerné, et peut donc attirer l'attention des usagers lors de leur navigation, il est également recensé automatiquement dans une page spécifique dédiée à la transgression constatée. La communauté est ainsi susceptible de se pencher particulièrement sur ces pages pour améliorer la qualité de l'information là précisément où des manquements ont pu être constatés.

Enfin, la communauté a décidé de proposer également une distinction positive aux articles particulièrement aboutis d'un point de vue encyclopédique, et qui respectent également scrupuleusement l'esprit wikipédien des piliers fondateurs. C'est ainsi qu'a vu le jour un bandeau destiné à signaler ces articles d'exception, remplacé dès 2005 par une procédure plus mâ̂trisée du fait d'abus de pose ou de retrait du bandeau par des contributeurs souvent inexpérimentés Cardon et Levrel (2009). Le label est décerné suite à une proposition d'un contributeur identifié et actif, et sur vote positif d'une part suffisante de la communauté, et peut être retiré via une procédure similaire. Les contenus « de qualité » (le niveau de distinction le plus élevé) est signalé sur la page distinguée par une étoile dorée, et sur une page recensant les contenus de qualité. Les «bons » contenus sont accordés de la même manière, mais les critères de respect des règles sont moins stricts. Signalés par une étoile argentée, les «bons » contenus sont également listés sur une page de l'encyclopédie dédiée, et peuvent le cas échéant perdre leur label. La communauté wikipédienne espère ainsi promouvoir certains contenus, et inciter les contributeurs à poursuivre leur effort.

\subsection{Collaborer, communiquer}

Mais si la création de ces divers moyens de signalement a bel et bien favorisé la participation de la communauté à l'encyclopédie, la collaboration n'y a pas forcément trouvé son compte. En effet, une partie importante du travail communautaire réside dans la possibilité d'échanger sur le sujet qui intéresse les contributeurs. Or le projet initial prévoit un seul espace d'échange entre contributeurs, dans la page de discussion liée à chaque article. Il est ainsi possible de discuter librement et publiquement du sujet traité dans l'article lié. Toutefois, ce canal de communication n'est généralement utilisé que dans les cas où des oppositions se sont manifestées au cours de la collaboration au point que l'intervention d'une troisième partie

10. http://fr.wikipedia.org/wiki/Wikipédia:Le_Bistro

11. http://fr.wikipedia.org/wiki/Wikipédia:AV. s'est révélée nécessaire pour éviter une guerre d'édition Stvilia et al. (2008). On constate d'ailleurs qu'une discussion ne s'est réellement amorcée via ce canal que dans un peu plus de $10 \%$ des articles de Wikipédia Jacquemin et al. (2008).

Les contributeurs ont en effet préféré rapidement exploiter d'autres possibilités de l'outil wiki pour assurer leurs échanges. Dès les débuts du projet, une page appelée le « Bistro » a ainsi été créée, qui donne accès quotidiennement aux différents sujets traités du jour, et permet d'en ajouter de nouveaux. La démarche est clairement informelle, et se revendique comme « un endroit pour se détendre, discuter du projet et se renseigner $» 10$. De nombreux contributeurs ont ainsi pris l'habitude de discuter sur ces pages des sujets les plus divers, concernant un article et souvent plusieurs - ce qui ne peut rentrer dans le cadre des pages de discussion des articles - mais également de l'administration de Wikipédia, voire de sujets d'actualités ou d'autres encore. Progressivement, d'autres espaces ont été créés pour répondre à des besoins particuliers en communication informelle, mais sur des thématiques spécifiques. On a ainsi vu poindre le « Bistro des nonfrancophones » pour les contributeurs parlant mal le français, «Legifer » pour discuter des problèmes légaux posés par les contenus de Wikipédia, etc. En 2009, un portail «Avenue des cafés et bistros 11 》 est créé, qui fédère, très officiellement cette fois, les différents espaces de discussion sur Wikipédia.

Enfin, la communauté a exploité un dernier espace de discussion offert par le wiki pour établir une communication plus personnelle entre ses membres, mais qui n'est possible qu'en direction de contributeurs régulièrement inscrits. En effet, le fonctionnement classique d'un wiki est de fournir à ses utilisateurs réguliers, et donc inscrits, la possibilité d'éditer et de mettre en ligne des pages web, et de participer à l'ensemble du projet. Dans Wikipédia, cet impératif d'inscription n’a pas été retenu dans le cadre de l'édition. Toutefois, lorsqu'un contributeur s'inscrit de manière à être identifié dans le projet, son identité wikipédienne est associée à une page qui lui appartient, et sur laquelle il peut déposer toutes les informations qui lui sont personnelles. Bien entendu, cette page est assortie d'une page de discussion, que les utilisateurs réguliers de Wikipédia utilisent fréquemment pour discuter de personne à personne, même si ces discussions sont publiques. De cette manière, les membres de la communauté contributrice ont trouvé une méthode pour amoindrir l'aspect «forum » des autres procédures de communication mises en place.

\subsection{Pour résoudre le conflit}

Malgré une réglementation simple et l'évidente bonne volonté d'un grand nombre de contributeurs sans laquelle l'encyclopédie n'aurait pu connaître un tel essor, la collaboration wikipédienne, qui consiste pour partie à confronter dans un même texte des points de vue totalement opposés, amène souvent des désaccords entre tenants d'opinions différentes. Comme nous l'avons vu ci-avant, la résolution du désaccord est envisagée essentiellement par l'échange d'arguments dans la page de discussion liée à l'article concerné. Tou- 
tefois, outre le fait que les possibilités de cette page sont peu exploitées, il est également possible et même fréquent que les désaccords fassent suite à une discussion dans un des autres espaces d'échange mis progressivement en place dans l'encyclopédie, comme le Bistro Viégas et al. (2004); Bryant et al. (2005); Auray et al. (2009). De nouveau, ce sont des initiatives de contributeurs sortant du chemin tracé qui ont suscité des moyens de résoudre le problème.

En effet, lorsqu'un désaccord se manifeste dans un espace de Wikipédia (souvent manifesté par des attaques ad hominem sur un espace d'échange, une activité particulièrement intense sur un article, ou encore une utilisation systématique du revert, une fonctionnalité du wiki qui permet de revenir à un état de la page à un moment déterminé en éliminant les contributions ultérieures), des contributeurs expérimentés ont commencé à s'entremettre entre les opposants pour tenter d'apaiser les tensions. Rapidement, cette initiative s'est standardisée : ces contributeurs diplomates se sont appelés « wikipompiers », chargés de détecter et d'éteindre les débuts de conflits (« wikifeux ») là où ils se déclaraient. Enfin, le rôle et la fonction ont évolué vers une procédure réglementaire de l'encyclopédie. Structurée dans l'espace de médiation, cette procédure implique des médiateurs élus par la communauté suite à une candidature, et le rôle des médiateurs consiste exclusivement à tenter de renouer un dialogue, idéalement serein, entre contributeurs en désaccord qui en font la demande.

Mais quand le conflit est avéré, que les attaques deviennent plus personnelles que scientifiques et que les efforts de médiation ne parviennent plus à juguler les transgressions des réglementations ou des principes fondateurs de Wikipédia, la communauté contributrice a souhaité que soit mise en place une instance capable de juger le conflit et surtout habilitée à prendre des décisions pour protéger l'encyclopédie elle-même. C'est ainsi qu'est né le "Comité d'arbitrage »12, chargé de traiter les plaintes déposées par des contributeurs entrés en conflit, attendu que toutes les possibilités de médiation ont été épuisées Viégas et al. 2007); Zlatic et al. 2006). Composé de sept, puis dix contributeurs réguliers élus pour une période de six mois 13 , le Comité d'arbitrage étudie la recevabilité des plaintes au regard des règles de Wikipédia, analyse les faits et les arguments des protagonistes et apporte des solutions au conflit, lesquelles incluent des sanctions de blocage allant d'une interdiction provisoire de contribuer à un article jusqu'au bannissement complet et définitif du projet. La démarche de l'arbitrage, initiée dans Wikipédia en 2005 (soit quatre ans après sa fondation), a évolué au cours du temps pour parvenir à la procédure relativement aboutie actuelle. Les arbitres ont notamment dû établir leur rôle, détaché de toute considération éditoriale et donc des contenus des contributions pour se consacrer au strict respect des piliers fondateurs de Wikipédia ainsi qu'à l'application des règlements édictés par la communauté des contributeurs.
Les débats qui précèdent chaque décision (recevabilité, proposition d'arrêt) sont publics, et les décisions elles-mêmes sont collégiales et doivent atteindre au consensus, comme c'est la philosophie dans l'encyclopédie elle-même Jacquemin (2010). Les décisions prises consistent généralement en un rappel ou une explicitation des règles et principes de Wikipédia assorti d'une protection technique plus ou moins longue de l'encyclopédie face à une menace.

\section{Démocratie et gouvernance dans le conflit}

\subsection{Le Comité d'arbitrage dans le dispositif?}

Apparu dans Wikipédia en 2005, plus de quatre ans après le début du projet, à l'instigation de la communauté wikipédienne 14, le Comité d'arbitrage est un bon exemple de démarche novatrice de la part des contributeurs de Wikipédia, à la fois iconoclaste et respectueuse du dispositif. Sortant complètement des schémas d'interaction établis à l'origine par les initiateurs du projet, le Comité d'arbitrage constitue dès sa création un archétype de transgression du fonctionnement de Wikipédia en tant que dispositif. En effet, au même titre que les autres propositions de la communauté que nous avons passées en revue, la création du Comité d'arbitrage résulte d'une part d'une lacune déplorée par la communauté dans la structure sociale mise en place dans Wikipédia, et de l'autre d'une consultation démocratique de la même communauté pour intégrer dans les procédures légitimes de l'organisation sociale wikipédienne cette proposition d'instance pour subvenir au défaut. Comme pour les autres procédures impliquées, on assiste dès lors à une tension entre deux types de comportements affichés dans la communauté : tantôt la poursuite de la finalité du dispositif amène des contributeurs à proposer des démarches qui sortent du cadre défini par le même dispositif en proposant, tantôt des contributeurs tentent d'intégrer ces démarches au cadre défini en les légitimant à travers des procédures licites eu égard à l'économie initiale du dispositif.

Cependant, le Comité d'arbitrage revêt une spécificité remarquable au regard des autres démarches étudiées. En effet, sa légitimation par une proposition soumise au vote de la communauté, et avalisée par un résultat positif, institue une disposition qui va à l'encontre d'un respect strict des piliers fondateurs ainsi que de la philosophie initiatrice de Wikipédia. En effet, la désignation d'un ensemble de contributeurs en tant qu'arbitres, même pour une durée limitée, confère à ces contributeurs un statut particulier, ce qui contrevient au principe d'égalité entre tous les participants au projet. Bien sûr, le statut d'arbitre n'est pas le seul à exister dans l'encyclopédie, et différents rôles existent qui distinguent certains participants des autres. Nous

12. Créé le 24 octobre 2004 par vote favorable de la communauté au cours d'un scrutin qui a rassemblé...61 votants (52 votes favorables, soit 85,24\% des avis exprimés), http://fr.wikipedia.org/wiki/Wikipédia:Comité_d'arbitrage_(vote)

13. Le quatorzième Comité d'arbitrage a été élu le 30 mars 2011 et seuls neuf des douze candidats ont été élus (un scrutin par candidat). En moyenne, 80,66 votes ont été exprimés par scrutin, et les arbitres désignés ont été élus par 60,66 électeurs, http://fr.wikipedia.org/wiki/Wikipédia:Comité_d'arbitrage/élection_d'arbitres_pour_le_14e_CAr

14. Dans la Wikipedia initiale, Jimmy Wales, co-fondateur du projet, est à l'origine de la création d'un Arbitration Committee dont le fonctionnement est assez proche des tribunaux avec jury populaire des États-Unis. Le fonctionnement du Comité d'arbitrage de la Wikipédia francophone, décrit précédemment, s'en distingue sur de nombreux points. 
avons ainsi signalé l'existence d'administrateurs et de médiateurs. Les premiers remplissent des tâches techniques et administratives dans l'encyclopédie, comme bloquer les adresses IP de vandales, bloquer, supprimer ou renommer des pages selon les décisions de la communauté (et souvent à la suite de conflits entre contributeurs). Les médiateurs, comme nous l'avons dit, sont chargés par la communauté de renouer le dialogue entre co-contributeurs en conflit en assurant une position d'intermédiaire dans la discussion. On notera encore le rôle de bureaucrate, dont les prérogatives sont essentiellement liées à l'attribution de statuts (administrateur, bureaucrate) - et donc l'accès à des outils techniques et administratifs - à d'autres utilisateurs sous le contrôle de la communauté, en plus du pouvoir de renommer les pages d'utilisateurs lorsque ceux-ci désirent changer d'identité électronique.

Le rôle d'arbitre se distingue toutefois de ces rôles essentiellement administratifs car aucun de ces utilisateurs « à statut » n'est fondé à prendre une décision qui engage un autre utilisateur, au contraire du statut d'arbitre. En effet, la tâche des arbitres a cela de spécifique qu'elle consiste à prendre - collégialement il est vrai - des mesures à l'encontre des contributeurs à Wikipédia qui sont entrés en conflit et n'ont pu résoudre leur différend grâce au panel de moyens d'apaisement des tensions offerts par la communauté wikipédienne. Ces mesures privilégiant la protection de l'encyclopédie face à toute menace, elles consistent généralement en une limitation partielle ou totale, provisoire ou permanente, de participation au projet. Un arbitre a donc un pouvoir de décision qui touche directement la liberté d'action d'autres participants au projet Wikipédia. Et si un tel pouvoir, unique dans le dispositif ${ }^{15}$, s'appuie sur les mêmes processus que les précédentes procédures, d'abord la proposition à la communauté et ensuite le vote démocratique permettant de l'avaliser, on peut toutefois s'interroger sur la légitimité effective du statut, et partant sur la prépondérance que confère ce statut.

\subsection{Le processus d'élection des ar- bitres}

Le déroulement-même du processus de désignation des arbitres pose également question. En effet, bien que désignés sous l'appellation d' "élection d'arbitres pour le $n^{\text {e }}$ Comité d'arbitrage », les principes liés à cette consultation sont très différents des scrutins que nous connaissons dans nos démocraties occidentales. Par exemple, la valeur des voix des électeurs reste très relative, chaque électeur ayant la possibilité de s'exprimer plusieurs fois au cours du même scrutin et d'accorder ainsi une voix à autant de candidats qu'il le désire, alors même que ces candidats sont rivaux, le nombre de postes d'arbitres étant limité. L'expression du choix des électeurs s'effectue de manière positive, négative ou neutre 16 , et de ce fait l'élection se rapproche plutôt d'une démarche de référendum. En effet, la candidature se présente plutôt comme une proposition dichotomique face à laquelle l'avis de la communauté est demandé : "Souhaitez-vous que le candidat untel devienne arbitre? ». Toutefois, l'accession au statut d'arbitre pour un candidat n'est pas seulement liée au assentiment obtenu ou non auprès de la communauté électrice à l'issue de ce référendum, mais elle dépend également des résultats obtenus par les autres candidats. Il s'agit d'abord d'atteindre plus de dix votes positifs, et dans tous les cas de recueillir deux fois plus d'avis positifs que négatifs (deux tiers des votes valides non neutres doivent être positifs). Mais comme le nombre d'arbitres dans le Comité d'arbitrage est limité (d'abord sept arbitres, puis dix à partir du troisième Comité d'arbitrage), obtiendront le statut convoité, parmi ceux qui réunissent les deux conditions sus-citées, les sept (ou dix) candidats dont la différence entre les voix positives et les voix négatives sera la plus élevée. À partir du deuxième Comité d'arbitrage, les candidats qui remplissent les conditions d'éligibilité mais ont obtenu un score trop bas pour être élus obtiennent le statut de « suppléants », et son appelés à siéger comme arbitres, dans l'ordre décroissant des scores de popularité, en cas de défection d'arbitres désignés, de manière à éviter autant que possible des élections partielles. Il faut également noter qu'il n'y a pas de « circonscription électorale », et que cette élection se joue en un seul tour, dont la durée varie entre les différents scrutins (mais ne peut être inférieure à huit jours).

D'autre part, la notion de liste électorale n'existe pas vraiment, ce qui n'est pas sans conséquence. Le vote est ouvert à tout membre de la communauté, mais pour éviter les abus et les maladresses, seuls les contributeurs ayant un minimum d'expérience 17 sont autorisés à voter. Dès lors, seuls les contributeurs régulièrement inscrits auront la possibilité de s'exprimer au cours d'un scrutin, car le nombre de contributions n'est vérifiable que lorsque les opérations sont effectuées par un utilisateur identifié. En outre, la consultation ellemême ne pourra se tenir à bulletin (électronique) secret, puisque la communauté est garante de la bonne tenue du scrutin et doit pouvoir vérifier la validité de chaque vote exprimé au regard du règlement qui l'encadre. En conséquence, des électeurs identifiés peuvent s'exprimer librement, mais sous le regard de la communauté, accepter ou refuser la candidature de chacun des candidats au cours de chaque élection. Le vote de neutralité, bien que recensé et comptabilisé au même titre que les votes positifs et négatifs, n'entre dans aucun calcul et n'influence en rien l'élection ou le rejet des candidats. Comme tel, le processus de désignation

15. À l'exception notable de Jimmy Wales, fondateur de l'Encyclopédie ainsi que de la Wikimedia Foundation Inc., association sans but lucratif chargée de développer le logiciel MediaWiki, à la fois plateforme technique et hébergeur de Wikipedia et des projets qui y sont reliés (Wiktionary, Wikiquote, Wikibooks, Wikimedia Commons, Wikisource, Wikispecies, Wikinews et Wikiversity), dont il est le président d'honneur. Dans l'instance anglophone de Wikipedia, Jimmy Wales dispose d'un pouvoir important sur le Comité d'arbitrage (Arbitration Committee), puisqu'il désigne lui-même les arbitres parmi les candidats que la communauté a gratifiés de plus de $50 \%$ de votes favorables. En outre, Jimmy Wales dispose d'une voix permanente dans ce Comité anglophone, qui est décisive en cas d'égalité entre les avis des autres arbitres (tie break). Jimmy Wales dispose encore de deux privilèges remarquables : celui de grâce (clemency) et celui de dissolution du Comité d'arbitrage. Toutefois, il n'y a pas d'exemple d'un abus que Jimmy Wales aurait fait de ces prérogatives.

16. Le choix de neutralité est comptabilisé et constitue une pratique largement répandue à travers tout le processus électoral du Comité d'arbitrage.

17. Durant les cinq premiers scrutins, pouvaient être électeurs tous les contributeurs qui avaient un minimum de 50 faits d'édition dans l'espace encyclopédique. Lors de la sixième élection, le nombre d'édits minimum est passé à 350 . 
des arbitres, qui tient à la fois du plébiscite et du référendum, est très éloigné des élections démocratiques auxquelles nos institutions nous ont habitués.

\subsection{Un corpus d'étude pour le Co- mité d'arbitrage}

Cependant, la mise en cause de la légitimité du statut d'arbitre, et donc du Comité d'arbitrage, ne peut aller de soi. Notre questionnement à ce sujet ne s'appuie en effet que sur une certaine originalité des prérogatives des arbitres à l'égard d'autres utilisateurs, qui irait à l'encontre de l'économie fondatrice du dispositif qui pourtant intègre ces arbitres. Il demande cependant à être corroboré par des faits observables et objectifs. Pour appuyer notre réflexion, il nous a donc paru indispensable d'analyser en profondeur le processus qui préside à la désignation des arbitres, investis dès lors de ces prérogatives.

En conformité avec la politique de libre accès à l'information du projet Wikipédia, concrétisée par des contenus librement exploitables et modifiables selon les termes de la licence Creative Commons CC-BY-SA, la Wikimedia Foundation Inc. met à disposition du public, deux fois par mois environ, une sauvegarde de l'ensemble des données textuelles de chaque instance de l'encyclopédie. Cette sauvegarde, librement et facilement téléchargeable ${ }^{18}$, se présente sous la forme d'un fichier unique compressé qui contient du texte balisé selon le langage XML, ce qui permet de l'intégrer dans un système de gestion de bases de données, en l'occurrence MySQL. Outre l'état actuel des articles de l'encyclopédie, cette sauvegarde comporte également l'historique complet de ces articles, les pages de discussion qui y sont associées, les pages personnelles des utilisateurs et leur historique, ainsi que l'ensemble du matériel « administratif » de l'encyclopédie : conseils et procédures, portails de gestion des bandeaux distinctifs etc. Parmi ces pages d'administration, on trouve notamment, pour le cas qui nous intéresse, l'ensemble de ce qui a été diffusé sur Wikipédia concernant la résolution du conflit, y compris le Comité d'arbitrage. Comme nous disposons de la sauvegarde réalisée le 24 avril 2011, nous avons accès aux archives des élections des quatorze Comités d'arbitrage qui ont siégé à la date de rédaction de cet article 19 .

À partir de cette énorme masse d'information, nous avons constitué un corpus de travail principal qui rassemble les données relatives au déroulement de chacune des élections au Comité d'arbitrage. Pour chacun des scrutins, les archives des élections au Comité d'ar- bitrage comportent notamment les dates de début et de fin de la période de vote, le nombre de postes d'arbitre à pourvoir, la durée du mandat en cas de succès et, pour chaque candidat, la liste des votants assortis des date et heure de leur vote, classés selon leur choix, qui peut être positif, négatif ou neutre ${ }^{20}$. Ces archives fournissent également le résultat final de l'élection à travers une liste d'arbitres, de suppléants et de candidats rejetés. Nous pouvons dès lors identifier l'ensemble des candidats au poste d'arbitre et l'accueil que leur candidature a reçu de la part de la communauté, l'ensemble des électeurs et la position qu'ils ont exprimée face à chaque candidature. Lorsqu'un candidat s'est retiré avant la fin de l'élection, nous avons traité sa candidature comme celle des autres si des votes ont eu le temps d'être exprimés, et nous avons éliminé cette candidature dans le cas contraire, en tenant plus compte de l'expression des votants qui représentent la communauté que de celle du candidat qui ne représente que lui-même. Nous n'avons pas tenu compte de certains votes exprimés qui ont été rayés par les électeurs qui les avaient exprimés car dans la plupart des cas il s'agissait d'un changement d'avis, un nouveau vote étant exprimé dans une autre rubrique de choix. Enfin, pour être valides, les votes doivent être exprimés par des contributeurs identifiés et expérimentés (350 opérations d'édition dans l'encyclopédie). Certains votes qui ne répondaient pas à ces conditions ont été exprimés, puis annulés. Nous ne les avons donc pas pris en compte. Par ailleurs, lorsqu'un votant a utilisé plusieurs identifications au long de l'existence du Comité d'arbitrage et qu'il a exprimé des votes sous ses différents identités 21 , nous avons autant que possible confondu les différentes identifications de manière à ne considérer qu'un seul votant.

Les corpus auxiliaires ont quant à eux été constitués à partir des résultats de deux autres types de candidatures de contributeurs à un rôle particulier dans l'encyclopédie. Il s'agit des rôles d'administrateur et de bureaucrate, qui présentent l'un et l'autre une particularité cruciale en commun avec le statut d'arbitre : tous bénéficient, pour effectuer les tâches qui leurs sont dévolues, de droits étendus par rapport à ceux du contributeur normal. Cependant, ni les administrateurs ni les arbitres n'ont de liberté d'initiative, et ils ne peuvent exercer leurs prérogatives particulières que suite à une décision régulière de la communauté ou du Comité d'arbitrage. Et donc, si ces deux rôles ont, comme celui d'arbitre, des privilèges qu'ils peuvent exercer à l'encontre d'autres utilisateurs, aucun d'eux ne dispose du pouvoir de décision d'utiliser ce pouvoir, contraire-

18. http://dumps.wikimedia.org/

19. Les élections du quinzième Comité d'arbitrage ont été clôturées le 30 septembre 2011 à minuit. Un nouveau Comité d'arbitrage a donc pris ses fonctions le $1^{\text {er }}$ octobre 2011.

20. Lors de l'élection des trois premiers Comités d'arbitrage, le choix entre ces trois positions n'était pas encore figé comme c'est devenu le cas pour l'élection partielle des troisièmes et quatrième Comités. La première élection consacrait trois postures : « Pour », "Contre » et «Abstention », cette dernière n'ayant pas de conséquence sur le résultat du vote. Lors de l'élection suivante, l'étiquette « Abstention » s'est dédoublée et les électeurs incertains et désireux néanmoins de s'exprimer pouvaient choisir entre « Neutre » et «Blanc » sans qu'il soit possible de distinguer une différence entre ces deux positions sans effet. La communauté wikipédienne s'est amusée de la multiplicité de ces votes sans effet, et certains d'entre eux ont été étiquetés «Violet », « Bleu », « Vert et jaune »... Lors de l'élection du troisième Comité d'arbitrage, c'est le terme « Neutre » qui s'est finalement imposé, malgré une survivance de la même plaisanterie : «Pleutre », «Feutre » ou « ertueN ». Étant donné que tous ces votes n'ont aucun impact sur l'élection ou le rejet du candidat, nous les avons assimilés à la position de neutralité qui s'est en définitive imposée.

21. De nombreux contributeurs à Wikipédia demandent à changer de nom d'utilisateur au cours du temps. On citera par exemple l'utilisateur Quoinonne qui apparaît à d'autres moments sous le nom de Briling ou d'Alençon, ou Caverna qui signe également Gemme et Glacier. Il ne s'agit pas d'alias, mais de changements de nom d'utilisateur demandés aux administrateurs de l'encyclopédie - en même temps que la page personnelle de ces utilisateurs est renommée à leur nouvelle identité - et ces utilisateurs n'utilisent jamais simultanément leurs différentes identifications : ce serait alors un comportement illicite appelé usage de « faux-nez ». 
ment aux arbitres. Nous remarquons également que la durée du mandat d'administrateur ou de bureaucrate n'est pas limitée dans le temps, à la différence de celui d'arbitre qui ne dure en principe que six mois, et au maximum un an dans les tâtonnements qui ont marqué les débuts du statut. Du fait du rapprochement naturel entre ces trois statuts privilégiés et malgré les distinctions de taille qui les séparent, il nous a semblé pleinement justifié d'étudier le déroulement du processus de désignation des arbitres sans nous affranchir d'un regard sur les deux autres rôles. Les deux corpus auxiliaires sont donc constitués l'un et l'autre de données collectées sur les pages relatives à l'élection respectivement des administrateurs et bureaucrates. Ils rassemblent la liste des contributeurs à Wikipédia qui ont proposé leur candidature assortie du résultat du vote et de la date de clôture de ce scrutin. Comme il ne s'agit pour nous que de données complémentaires, nous n'avons pas exploité l'information complète relative à ces élections (notamment la liste des électeurs et le vote exprimé).

Tout le matériel factuel collecté dans ces corpus a été traité de manière semi-automatique afin de rendre cohérentes les diverses données, et notamment la mise en forme des identifications - on trouve par exemple des votes du même électeur sous les signaturesP@d@w@ne et Padawane. Ce traitement a également contribué à présenter les données collectées sous une forme structurée, de manière à obtenir une identifi- cation par champ des types d'information, et surtout à insérer le tout dans un système de gestion base de données relationnelle. L'information collectée dans chacun de ces trois corpus est donc stockée dans une base de données MySQL, et chaque candidat est associé à un ou plusieurs scrutins, aux résultats de ces scrutins et aux électeurs qui s'y sont exprimés, eux-mêmes mis en relation avec les avis qu'ils ont émis.

\section{4 Électeurs, candidats et élus pour l'arbitrage}

$\mathrm{Au}$ cours de notre réflexion sur le phénomène Wikipédia et sur son évolution largement spontanée du fait de l'impulsion de ses contributeurs, nous avons été amené à l'observer à l'aune du concept de dispositif, qui semblait un prisme intéressant pour en apprécier à la fois la diversité et la cohérence. Au cours de cet examen, nous avons toutefois été conduit à questionner cette lecture, car Wikipédia semblait accepter et assimiler une institution qui va à l'encontre de la cohérence attendue. Nous avons donc extrait les données factuelles relatives à l'accès à cette institution de manière à disposer d'informations objectives pour approfondir notre examen. Toujours dans un souci d'objectivité, c'est à travers un examen purement quantitatif que nous avons choisi d'exploiter les informations que nos corpus contiennent.

\begin{tabular}{|rl|}
\hline 14 & Comités d'arbitrage différents \\
15 & élections (dont 2 partielles) \\
181 & candidatures \\
91 & postes à pourvoir \\
86 & candidatures approuvées \\
116 & candidats différents \\
57 & arbitres différents \\
\hline
\end{tabular}

TABLE 1 - Le Comité d'arbitrage : chiffres des scrutins

Pour appréhender le phénomène de la désignation des arbitres, nous devons passer par plusieurs phases d'analyse dont les premières seront simples et prépareront la dernière qui s'attachera à prendre en compte les différentes variables considérées lors de la constitution des corpus. Un premier niveau d'analyse, totalement unidimensionnel, permet d'évaluer les proportions du phénomène sous plusieurs angles, ceux des différents acteurs de l'élection : le Comité d'arbitrage qui intègre les nouveaux arbitres, les candidats et les électeurs. Ainsi, dans le tableau 1 nous avons un aperçu global de ce que l'élection apporte au Comité d'arbitrage. On constate d'abord qu'avec à peine 12 candidatures en moyenne par élection - alors même qu'un collège d'arbitres complet est de sept jusqu'au troisième Comité d'arbitrage, puis de dix ensuite - la concurrence n'est pas très importante. Avec 86 succès électoraux seulement pour 14 collèges d'arbitres différents, l'en- semble des postes (91 en tout, avec les élections partielles) n'a évidemment pas pu être pourvu. Nous avons insisté sur les particularités des conditions à remplir pour être élu arbitre, et notamment l'exigence d'obtenir deux fois plus de votes positifs que de négatifs au moins. Cette règle trouve ici sa raison d'être et sa justification : il ne s'agit pas de donner le statut d'arbitre et les pouvoirs afférant à un contributeur repoussé par la communauté. On peut également constater une certaine continuité dans l'appartenance au collège des arbitres. En effet, sur l'ensemble des candidatures ayant rencontré le succès, un tiers sanctionne la candidature d'un ancien arbitre, ce qui veut dire qu'un arbitre sur deux est élu à nouveau au comité d'arbitrage. Sans que ces chiffres puissent être considérés comme choquants, ils tendent à montrer une certaine pérennité dans le statut d'arbitre. 


\begin{tabular}{|rl|rl|}
\hline \multicolumn{2}{|c|}{ Administrateurs } & \multicolumn{2}{c|}{ Bureaucrates } \\
\hline \hline 402 & candidatures & 20 & candidatures \\
296 & élus & 12 & élus (tous administrateurs) \\
77 & rejetés & 5 & rejetés (4 administrateurs) \\
29 & abandons & 3 & abandons (tous administrateurs) \\
\hline
\end{tabular}

TABLE 2 - Résultats des élections d'administrateurs et de bureaucrates

Les tableaux suivants nous permettent d'apprécier les grandeurs relatives aux candidatures, d'abord pour les rôles d'administrateur et de bureaucrate (tableau 2), ensuite pour le statut d'arbitre (tableau 3. Notre optique initiale était, lorsque nous avons constitué nos corpus secondaires relatifs à l'élection des administrateurs et des bureaucrates, de mettre en perspective d'une part les modalités d'accès au statut d'arbitre, et de l'autre le comportement des autres élus de l'encyclopédie dans cette élection. Cependant, au regard du tableau2 l'exploitation des données portant sur l'élection des bureaucrates nous a semblé moins pertinente. En effet, les tendances de comportement d'une population aussi faible ne pourraient être significatives et les conclusions qu'on en tirerait resteraient douteuses.
De plus, l'ensemble des bureaucrates - et même pratiquement tous les candidats malheureux à ce rôle - font partie des administrateurs. Comme notre objet d'étude concerne l'élection des arbitres et l'impact que le comportement d'autres élus aux droits étendus a sur cette élection, nous avons résolu d'abandonner les données concernant les bureaucrates, nous refusant à distinguer deux groupes de wikipédiens particuliers là où une des catégories est incluse dans l'autre. Par contre, avec plus de quatre cents candidatures, dont $75 \%$ couronnées de succès et près de 80 candidats rejetés par la communauté, il est possible de détecter des tendances de comportements significatives liées à la population entière, mais aussi à des sous-groupes relativement nombreux.

\begin{tabular}{|c|c|c|c|}
\hline \multicolumn{2}{|r|}{ Candidatures } & \multicolumn{2}{|c|}{ Candidats (différents) } \\
\hline 181 & candidatures & 116 & candidats \\
\hline 86 & succès & 57 & arbitres \\
\hline 53 & échecs (rejet) & 43 & rejetés \\
\hline 69 & candidatures d'admin. & 40 & candidats admin. \\
\hline 42 & succès d'admin. & 28 & arbitres admin. \\
\hline 11 & échecs d'admin. (rejet) & 10 & rejetés admin. \\
\hline 0 & candidature de rejeté admin. & 0 & candidat rejeté admin. \\
\hline
\end{tabular}

TABle 3 - Résultats des élections d'arbitres

En observant le tableau 3 nous pouvons apprécier la manière dont dont la communauté réagit aux candidatures au Comité d'arbitrage. D'un point de vue général, on peut voir que la communauté est plutôt bienveillante envers les candidats, puisque les échecs sont très minoritaires. Moins de $30 \%$ des candidatures sont rejetées, et près de deux candidats sur trois obtiennent plus des deux tiers de votes positifs nécessaires pour être éligibles. Sans tenir compte des candidats éligibles mais qui ne sont pas élus ${ }^{22}$, les candidats élus au statut d'arbitres dépassent les candidats rejetés de $25 \%$ (et les candidatures couronnées de succès dominent les candidatures rejetées de près de $40 \%$ ). Ces résultats dénotent plus une tendance des électeurs à saluer l'initiative de leurs pairs pour gérer le conflit que de la défiance à l'endroit de dépositaires d'un pouvoir particulier.

C'est toutefois dans la partie basse de ce tableau 3 que les enseignements de l'information portant sur les candidats et leurs candidatures sont les plus parlants. En effet, les chiffres qui concernent les candidatures de contributeurs qui remplissent déjà un rôle d'admi- nistrateur sont encore plus tranchés, et pleins d'enseignements. On constatera d'abord que près de $40 \%$ des candidatures déposées le sont par un administrateur, et ces derniers représentent également $35 \%$ de l'ensemble des candidats. Plus impressionnant encore, $60 \%$ des candidatures d'administrateurs sont couronnées de succès, contre $47 \%$ pour l'ensemble des candidatures. On constate même qu'un candidat précédemment élu administrateur a $70 \%$ de chances d'être élu, alors que la moyenne est de moins de $50 \%$. La communauté semble de plus répugner à jeter l'anathème sur ses administrateurs : rares sont ceux qui ont été rejetés, avec 11 candidatures (et 10 candidats) repoussées seulement sur les 15 scrutins, soit cinq fois moins que pour l'ensemble des candidatures. On notera également que lorsqu'un candidat a été rejeté comme administrateur, il semble se désintéresser de la gouvernance du projet, en tout cas via le Comité d'arbitrage, puisqu'il n'y a pas d'exemple d'un wikipédien n'ayant pas réussi à intégrer les administrateurs qui se soit présenté pour devenir arbitre. 


\begin{tabular}{|c|c|c|}
\hline Votes (nombre de voix) & Électeurs & Moyenne \\
\hline 11063 votes exprimés & 592 électeurs différents & 18,69 \\
\hline 6489 votes positifs & 564 électeurs votant oui & 11,51 \\
\hline votes négatifs & électeurs votant non & 5,61 \\
\hline votes neutres & électeurs votant neutre & 7,23 \\
\hline
\end{tabular}

TABLE 4 - Votes et électeurs au long des 13 Comités d'arbitrage différents

Un aperçu enfin du simple relevé de la distribution des électeurs ( $c f$. tableau 4) permet de conforter certaines de nos observations précédentes. Tout d'abord, plus de la moitié des votes exprimés sont positifs, ce qui appuie le constat selon lequel les électeurs sont plutôt bien disposés à l'égard des candidats à un statut d'arbitre. Cette complaisance est en outre confirmée par un niveau relativement similaire de votes neutres et de votes négatifs. Le vote neutre n'ayant aucun im- pact sur le vote, cette similarité tendrait à montrer que les électeurs ont une propension à éviter les décisions frontales, et choisissent aussi facilement une sage neutralité, « gaspillant » ainsi leur voix, que l'opposition directe. La répartition des types de votes par électeurs différents semble toutefois tempérer ce constat, les électeur ayant choisi la neutralité étant sensiblement moins nombreux que ceux qui ont dans un cas au moins choisi l'opposition.

\begin{tabular}{|llrrrr|}
\hline Scrutin & Date & Inscrits & Candidats & Votes & Électeurs \\
\hline \hline 1 & Mars 2005 & 2698 & 12 & 397 & 57 \\
1bis & Juin 2005 & 3730 & 4 & 106 & 36 \\
2 & Sept. 2005 & 5068 & 13 & 464 & 64 \\
3 & Mars 2006 & 8986 & 16 & 1092 & 121 \\
$3-4$ & Juin 2006 & 11743 & 10 & 525 & 85 \\
$4-5$ & Sept. 2006 & 14414 & 14 & 683 & 87 \\
$5-6$ & Mars 2007 & 20105 & 16 & 882 & 111 \\
$6-7$ & Sept. 2007 & 25655 & 12 & 653 & 102 \\
$7-8$ & Mars 2008 & 31908 & 13 & 955 & 142 \\
$8-9$ & Sept. 2008 & 37921 & 13 & 1092 & 146 \\
$9-10$ & Mars 2009 & 43867 & 14 & 976 & 129 \\
$10-11$ & Sept. 2009 & 49626 & 12 & 777 & 119 \\
$11-12$ & Mars 2010 & 55528 & 10 & 813 & 125 \\
13 & Sept. 2010 & 60963 & 10 & 754 & 116 \\
14 & Mars 2011 & 66272 & 12 & 968 & 126 \\
\hline
\end{tabular}

TABle 5 - Évolution comparée du Comité d'arbitrage et des wikipédiens

Finalement, le tableau 5 nous permet d'appréhender une partie de ces mêmes données factuelles simples dans leur évolution à travers les différents scrutins au Comité d'arbitrage. Le constat le plus marquant et le plus important réside dans la faible représentativité de plus décroissante au cours du temps - de l'électorat du Comité d'arbitrage au regard des inscrits de l'Encyclopédie. Ne représentant que $2 \%$ des inscrits lors de la première élection, les électeurs ne font que perdre du terrain par rapport au nombre d'inscrits pour n'en représenter qu'à peine $0,2 \%$ lors de la dernière consultation, malgré une tendance réelle à devenir plus nom-

Il s'agit maintenant d'aborder des données plus complexes et qui prennent en considération plusieurs des facteurs des informations que nous avons collectées à partir de nos corpus de travail. Le tableau 6 nous a permis de croiser ces différents facteurs et de mettre en évidence plusieurs phénomènes qui viennent appuyer nos précédentes observations et étayer notre réflexion sur le fonctionnement du Comité d'arbitrage et son intégration dans le dispositif Wikipédia. Il nous breux en chiffres absolus. On constate également, de manière un peu plus indirecte, que les électeurs ont tendance à ne pas donner de voix (tous choix confondus) à chaque candidat : les électeurs n'accordent un vote qu'à environ $60 \%$ des candidats en moyenne. Là encore, on peut y voir la marque d'une attitude positive de la part de la frange des contributeurs qui participent aux élections. En effet, lorsqu'un électeur n'a pas d'avis sur un ou plusieurs des candidats lors d'un scrutin auquel il participe par ailleurs, il ne verra pas d'inconvénient à ne pas s'exprimer plutôt que de voter sans conviction.

permet notamment d'analyser la part que prennent les électeurs admis ou rejetés en tant qu'administrateurs dans l'élection ou dans l'éviction des candidatsarbitres. On peut d'abord constater que, quelle que soit la catégorie de candidat à l'arbitrage (candidat élu ou rejeté, candidat administrateur ou non), la part des voix issues d'électeurs qui remplissent le rôle d'administrateur est constante et importante à environ $45 \%$. Les électeurs qui ont vu rejetée leur candidature pour de- 


\begin{tabular}{|l|l|rr|rr|rr|rr|}
\cline { 3 - 10 } \multicolumn{2}{c|}{} & \multicolumn{2}{c|}{$\begin{array}{c}\text { Arbitres } \\
\text { + admin. }\end{array}$} & \multicolumn{2}{c|}{$\begin{array}{c}\text { Arbitres } \\
\text { pas admin. }\end{array}$} & \multicolumn{2}{c|}{$\begin{array}{c}\text { Admin rej. } \\
\text { arbitres }\end{array}$} & \multicolumn{2}{c|}{$\begin{array}{c}\text { Pas admin. } \\
\text { rej. arbitres }\end{array}$} \\
\cline { 3 - 11 } \multicolumn{2}{c|}{} & Voix & Élect. & Voix & Élect. & Voix & Élect. & Voix & Élect. \\
\hline \multirow{3}{*}{ Tous } & électeurs & 2491 & 467 & 2153 & 462 & 245 & 162 & 476 & 243 \\
& coutre & 254 & 110 & 298 & 122 & 286 & 168 & 1084 & 344 \\
& neutres & 322 & 145 & 418 & 165 & 140 & 90 & 708 & 222 \\
& total & 3067 & 493 & 2869 & 493 & 671 & 287 & 2268 & 450 \\
\hline \multirow{3}{*}{ Électeurs } & pour & 1192 & 170 & 961 & 163 & 87 & 60 & 195 & 86 \\
admin. & contre & 88 & 30 & 120 & 41 & 153 & 79 & 517 & 135 \\
& neutres & 144 & 58 & 208 & 77 & 56 & 37 & 325 & 96 \\
& total & 1424 & 172 & 1289 & 169 & 296 & 109 & 1037 & 158 \\
\hline \multirow{3}{*}{ Électeurs } & pour & 92 & 17 & 71 & 15 & 17 & 11 & 30 & 12 \\
rej. admin. & contre & 17 & 5 & 22 & 7 & 14 & 8 & 40 & 13 \\
& neutres & 11 & 5 & 17 & 7 & 2 & 2 & 16 & 8 \\
& total & 120 & 18 & 110 & 17 & 33 & 13 & 86 & 17 \\
\hline
\end{tabular}

TABLE 6 - Influence du statut d'administrateur sur l'élection des arbitres

venir administrateurs ont également une part de voix constante, à $5 \%$ à peine. C'est donc la moitié des votes exprimés pour le Comité d'arbitrage qui sont issus de contributeurs qui sont impliqués au niveau de l'administration - ou ont tenté de l'être, avec une surreprésentation importante de la part des administrateurs dans l'ensemble du scrutin. On note d'ailleurs des différences dans la manière donc les administrateurs expriment leur avis par rapport aux autres électeurs. En effet, les votes qu'ils accordent à leurs pairs administrateurs, qu'ils soient positifs ou négatifs, sont beaucoup plus tranchés que lorsqu'ils votent pour des candidats qui n'occupent pas de rôle particulier. Les choix des administrateurs entre eux sont également beaucoup plus radicaux que la tendance affichée par le comportement de l'ensemble des électeurs. En effet, alors que les votes négatifs portant sur la candidature d'administrateurs élus arbitres représentent $10 \%$ des votes positifs de la part de tous les électeurs, ils atteignent à peine $4,5 \%$ des votes positifs dès lors que les administrateurs seuls s'expriment. De plus, lorsque les administrateurs votent pour des candidats qui ne sont pas administrateurs mais vont pourtant être élus arbitres, leurs choix s'assimile à celui des autres électeurs, avec respectivement $12,4 \%$ et $13,8 \%$ de votes négatifs respectivement de la part des administrateurs et de l'ensemble des électeurs. Le même type de comportement plutôt intransigeant apparait également lorsqu'il s'agit de rejeter la candidature d'un confrère administrateur, avec des votes favorables qui ne représentent que $56 \%$ des votes d'opposition, alors que les votes positifs de l'ensemble des électeurs atteint $86 \%$ de leurs votes contre la candidature. Cette tendance à la fermeté est d'autant plus sensible que dans les deux cas de figure, le nombre de votes de neutralité est très nettement plus bas que dans les autres configurations. Par contre, leurs pratiques de vote face à des candidats non distingués par un rôle particulier montrent globalement les mêmes comportements que le reste de la communauté votante.

La tendance générale est, malgré un nombre non négligeable de votes contre des administrateurs, à considérer leurs candidatures sous un jour particulièrement positif. Outre les ratios pour/contre particulièrement favorables lorsqu'un administrateur est élu arbitre (plus de $81 \%$ en moyenne lorsqu'il s'agit d'un administrateur contre près de $75 \%$ pour les autres candidats, sachant qu'un candidat ne peut être élu qu'avec
$66 \%$ au moins et que ce sont les candidats aux meilleurs scores qui sont désignés), la différence reste suffisamment significative. Mais c'est surtout en creux que cette complaisance reste la plus évidente, avec une remarquable baisse du nombre de votes portant sur les candidats malheureux au Comité d'arbitrage lorsqu'ils sont administrateurs. On remarquera enfin, mais de manière plus marginale car la catégorie est peu nombreuse, que les électeurs rejetés en tant qu'administrateurs sont les seuls dont les votes négatifs sont toujours plus nombreux que les votes neutres, alors que la tendance est plutôt à un équilibre favorable à la neutralité.

À l'issue de cet examen, nous avons pu dégager plusieurs d'éléments sur la manière dont les arbitres sont désignés par la communauté. À l'appui de notre questionnement sur la légitimité de ce collège arbitral, nous avons d'abord constaté la faible représentativité de l'électorat des arbitres. Ensuite, nous avons constaté qu'un arbitre sur deux environ avait bénéficié de plus d'un mandat, ce qui tend à créer une forme de «personnel arbitral ». De plus, la représentation massive des administrateurs à la fois au sein des arbitres et parmi les électeurs des arbitres, alors que le rôle d'administrateur les fait déjà bénéficier de pouvoirs étendus, permet de voir dans un groupe relativement restreint un noyau de gouvernance dans le dispositif wikipédien, alors même que Wikipédia est basée sur une égalité des contributeurs et sur le principe d'une gouvernance de la communauté. On est dès lors fondé à s'interroger sur la pertinence, et même la validité du choix opéré lors de la régularisation du Comité d'arbitrage.

Pourtant, si la validité de la démarche de consultation n'est pas à mettre en cause, puisque l'ensemble de la communauté est invitée autant à donner son avis sur les initiatives ou les candidatures déposées qu'à faire des propositions ou à s'impliquer dans la gestion du projet, le déroulement du processus de légitimation peut quant à lui être questionné. En effet, malgré les nombreuses pages consacrées à l'explicitation des processus d'administration de Wikipédia, le fonctionnement communautaire du dispositif reste inconnu du plus grand nombre des wikipédiens, et il faut faire un effort sérieux pour appréhender la globalité de son organisation. En outre, les pages consacrées aux votes sont souvent d'un accès compliqué, et il faut en faire une veille régulière pour se tenir au courant des consul- 
tations en cours. Pourtant, la démarche de validation des décisions et des nomination à un statut particulier gagnerait en légitimité si la communauté était davantage représentée parmi les votants, ou même si elle était simplement informée qu'un scrutin est en cours et libre de choisir d'y participer ou non. Il serait dès lors profitable d'ajouter automatiquement sur les pages d'articles ${ }^{23}$ un encart signalant ces scrutins. Une telle initiative permettrait de réduire une disparité contestable, que nous avons notée entre les contributeurs très impliqués titulaires d'un statut particulier des autres.

\section{Conclusion : une gouver- nance spontanée}

Comme on a pu le voir, la collaboration, pour essentielle qu'elle soit à la construction de Wikipédia, est néanmoins très peu structurée ou explicitée dans les principes fondateurs de l'encyclopédie : seule une courtoisie de bon aloi est demandée aux participants, tandis que l'égalité de statut entre contributeurs n'est que sous-entendue. En tant que dispositif de collaboration, Wikipédia ne peut que bénéficier de cette latitude accordée volontairement par des règles simples et peu nombreuses, qui est de nature à favoriser son essor et une réelle créativité de Certeau (1980). De même, la communauté participante a bien intégré la finalité du projet, à savoir un mode de collaboration visant à la construction de connaissances encyclopédiques : prenant consciemment des initiatives en dehors du cadre prévu, les contributeurs négligent certaines pistes proposées (les pages de discussion) et se ménagent des espaces plus propices, dans leur pratique du dispositif, pour atteindre l'objectif visé Hert (1999).

Toutefois, et se rapprochant plus de la conception foucaldienne de dispositif Foucault et al. (1994); Peraya (1999), on voit poindre malgré cette liberté prise avec les principes de départ une réaffirmation de la notion de gouvernance, voire une expression d'un besoin de pouvoir, à la fois exercé et subi, quoique légitimé par un recours systématique à une validation de la communauté entendue comme un idéal démocratique Lebland (1999). En effet, on constate dans chaque apport de la communauté un besoin ou une émergence de réglementation, une définition de plus en plus précise, de plus en plus normalisée d'une procédure, une approbation nécessaire de la proposition par un vote de la communauté. L'initiative la plus informelle ellemême, le Bistro, se voit ainsi après quelques années mis en concurrence avec d'autres espaces, chaque type de conversation soi-disant informelle étant catégorisé et assigné réglementairement à un lieu précis de l'encyclopédie.

De manière beaucoup plus profonde, le Comité d'arbitrage constitue un exemple bien plus ambivalent d'une initiative de la communauté car il se développe explicitement sous l'égide de la communauté gouvernante. Or, nous avons vu l'importance qu'occupe dans l'arbitrage un groupe restreint de contributeurs qui concentrent les pouvoirs les plus vastes de l'ensemble du dispositif wikipédien, ce qui va à l'encontre des principes qui le fondent. Tout en revendiquant donc une réelle légitimité sur laquelle il s'appuie, le Comité d'arbitrage dans son fonctionnement sort donc complètement du cadre du dispositif dont il entend assurer l'intégrité. La loyauté de ses membres envers la finalité du dispositif lui permet néanmoins jusqu'à présent de s'intégrer sans heurts à l'économie générale de Wikipédia, mais l'équilibre demeure précaire entre loyalisme apparent et transgression profonde.

Entre deux visions très distinctes de la gouvernance du dispositif collaboratif Wikipédia, les contributeurs semblent opter pour celle qui privilégie les principes fondateurs, puisque chaque initiative originale pour gérer l'encyclopédie se voir rapidement formalisée, normalisée en une procédure presque administrative. Telle quelle, elle est confrontée à l'approbation de la communauté et finalement validée ou rejetée, auquel cas elle est bannie du dispositif. Pourtant, si dans la forme le pouvoir incombe bien à la communauté selon les principes démocratiques qui régissent Wikipédia, c'est dans les faits une part infime de la communauté qui s'exprime et décide de l'orientation et de la gouvernance du dispositif, tant pour l'adoption de procédures strictes que pour l'élection des contributeurs à statut particulier. En cela, l'expression du pouvoir dans le dispositif dépasse sans doute le clivage entre le loyalisme de Foucault et al. 1994) et le braconnage de de Certeau (1980) pour inventer une troisième voie, toujours dans l'entre deux qui caractérise la notion de dispositif Peeters et Charlier (1999).

\section{Références}

Auray, N., Hurault-Plantet, M., Poudat, C. et JacQUemin, B. (2009). La négociation des points de vue. une cartographie sociale des conflits et des querelles dans le wikipédia francophone. Réseaux, 154(2):1550 .

BARBE, L. (2009). La co-construction des informations et des savoirs sur le web participatif : étude par les acteurs. In Intelligence collective et organisation des connaissances. Actes du 7ème colloque du chapitre français de l'ISKO, pages 165-171, Lyon.

Bryant, S. L., Forte, A. et Bruckman, A. (2005). Becoming wikipedian : transformation of participation in a collaborative online encyclopedia. In Proceedings of the 2005 international ACM SIGGROUP conference on Supporting group work, pages 1-10, Sanibel Island, Florida, USA. ACM Press.

CArdon, D. et Levrel, J. (2009). La vigilance participative. une interprétation de la gouvernance de wikipédia. Réseaux, 154(2):53-89.

de Certeau, M. (1980). L'invention du quotidien. 1. Arts de faire. Gallimard, Paris.

Foucault, M., Defert, D., Ewald, F. et Lagrange, J. (1994). Dits et écrits, 1954-1988. 3, 1976-1979. Bibliothèque des sciences humaines. Gallimard, Paris.

HeRT, P. (1999). Internet comme dispositif hétérotopique. Hermès, 25:93-107.

23. Les informations de début de vote et de clôture de scrutin, mêlées à de multiples autres actualités - ce qui les rend d'un accès malaisé -, sont signalées sur la page d'accueil du portail encyclopédique. La majorité des usagers de l'encyclopédie accèdent à l'information non par le portail, mais par une page d'article. 
JacQuemin, B. (2010). Un dispositif de collaboration : l'exemple de wikipédia. In Appel, V., Boulanger, H. et MAssou, L., éditeurs : Les dispositifs d'information et de communication. Concept, usages et objets, pages 117-136. De Boek Université, Bruxelles, Louvain.

Jacquemin, B., Lauf, A., Poudat, C., HuraultPlantet, M. et Auray, N. (2008). Managing conflicts between users in wikipedia. In Flejter, D., GRzonkowski, S., KaczmareK, T., Kowalkiewicz, M., NAgle, T. et Parkes, J., éditeurs : Business Information Systems BIS 2008 Workshops Proceedings : Social Aspects of the Web SAW 2008, pages 81-93, Innsbruck. Poznań University of Economics.

Leblanc, G. (1999). Du déplacement des modalités de contrôle : Contrôle des représentations \& maîtrise du public. Hermès, 25:233-242.

Levrel, J. et CARdon, D. (2009). Contribuer et surveiller : l'autorégulation sur wikipédia. Documentaliste-Sciences de l'information, 46(1):5658.

Peeters, H. et Charlier, P. (1999). Contribution à une théorie du dispositif. Hermès, 25:15-23.
Peraya, D. (1999). Médiation et médiatisation : le campus virtuel. Hermès, 25:153-167.

Stvilia, B., Twidale, M. B., Smith, L. C. et Gasser, L. (2008). Information quality work organization in wikipedia. Fournal of the American Society for Information Science and Technology, 59(6):983-1001.

Viégas, F. B., Wattenberg, M. et Dave, K. (2004). Studying cooperation and conflict between authors with history flow visualizations. In Proceedings of the SIGCHI conference on Human factors in computing systems, pages 575-582, Vienne, Autriche.

Viégas, F. B., Wattenberg, M., Kriss, J. et Ham, F. V. (2007). Talk before you type : Coordination in wikipedia. In HICSS '07: Proceedings of the 40th Hawaii International Conference on System Sciences, page 78.

Zlatic, V., Bozicevic, M., Stefancic, H. et Domazet, M. (2006). Wikipedias : Collaborative web-based encyclopedias as complex networks. Physical Review E, 74(1):6-11. 